\title{
Research on the Concrete Crater Depth of Penetration by a Rigid Projectile
}

\author{
Qiaoheng Liang ${ }^{1, a}$, Luhui Yan ${ }^{1, b}$,Lingfeng $\mathrm{Li}^{2, \mathrm{c}}$ \\ ${ }^{1}$ Basic Education School of Commanding Officer, National University of Defense Technology, \\ Changsha 410072, China \\ ${ }^{2}$ Naval University of Engineering, Wuhan 430033, China \\ aqhafx@163.com, byan_I_h@aliyun.com.cn,'haochidehj94@163.com
}

Keywords: Rigid Projectile; penetration; concrete; crater depth; internal friction angle; strain rate

\begin{abstract}
Based on the theory of slip line and compared with the subject research of rigid stamping die press into semi-infinite rigid-plastic media, the crater depth formula of rigid-plastic projectile penetration concrete considering internal friction angle is established. Moreover, the relationship between the internal friction angle and impact velocity is researched by the Mohr-Coulomb theory and combined with the strength dynamic effect, and the conclusion in this paper is compared with the penetration test result of UHMWPE fiber reinforced concrete. The result shows that, the crater depth will be deeper as the internal friction angle increases and the internal friction angle will be larger with the increasing of the impact velocity.
\end{abstract}

\section{Introduction}

In the process of being impacted and penetrated by a rigid projectile with high speed, the concrete target will form a funnel crater because of the free-surface effect of concrete target which would lead to the material reverse jet of concrete surface. Academic studies show [1-3], the crater depth and projectile diameter are related, it can be expressed as $h=k d$, where $k$ is the coefficient of crater depth, and $k=2.0$ was suggested according to the tests data by Forrestal [1] and Frew [2]. Based on the theory of slip line, Li et al.'s [3] create the crater depth formula of a normal projectile impacted concrete target and recommend $k=0.707+H / d$. However, their analysis model only consider the slip line field of internal friction angle as $45^{\circ}$ and not consider the change of the angle, and it is inconsistent with the internal friction angle effect of concrete materials.

According to the test data, the fitted formula of crater depth $k=f_{c}^{-0.2056} V_{0}^{0.7539}$ was suggested in Ref. [4] which considering that the crater depth is associated with the concrete strength and projectile impact velocity. Due to lack of the mechanism basis, the applicability of the formula is still worth studying.

The crater depth theory of rigid-plastic projectile penetration concrete is further researched in the paper, and the slip line filed model considering the effect of internal friction angle is built, then the crater depth formula considering the internal friction angle is established. Moreover, based on the material strength dynamic effect, the relationship between the internal friction angle and impact velocity is studied. The result is compared with the penetration test result of UHMWPE fiber reinforced concrete, and it shows that the theoretical calculation is in good accordance with the test and the theory is correct and feasible in this paper.

\section{The Slip Line Filed Model Considering the Internal Friction Angle Effect}

\subsection{The slip line field}

2.1.1 The slip line field crater created by a flat-nose projectile

The problem of a flat-nose projectile impact on concrete can be compared with the subject research of rigid stamping die press into semi-infinite rigid-plastic media [5]. The quality of concrete is ignored in the paper, and it is assumed that near the impact point of the concrete reaches limit equilibrium state when the projection impact the concrete, then, the slip line filed considering 
internal friction angle is established and it is shown in Fig.1. In general, there are three regions in the flowing plastic zone. The $I$ region in Fig. 1 is the active region, and the angle between critical slip surface and horizontal plane is $\alpha^{\prime}=45^{\circ}+\varphi / 2(\varphi$ is the internal friction angle). The $I I$ region is transition region, whose curve boundaries are usually taken as logarithmic spiral lines and the formula is $r=r_{0} \exp (\theta \tan \varphi), \theta \in\left(0^{\circ}, 90^{\circ}\right)$. The $I I I$ region is passive region, whose boundaries are all straight lines, and the angle $\alpha=45^{\circ}-\varphi / 2$.

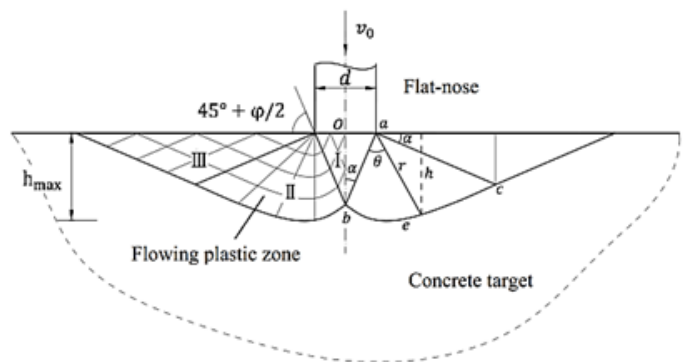

Fig.1 The slip line field of concrete crater created by a flat-nose projectile.

According to figure 1, and based on the logarithmic spiral formula $r_{0}=d /(2 \sin \alpha)$, the height $h$ of the slip surface $\overparen{\text { bec }}$ distance from the horizontal plane in geometry can be lead to

$$
h=\frac{d}{2 \sin \alpha} e^{\theta \tan \varphi} \sin \left(\frac{\pi}{2}-\theta+\alpha\right) \text {. }
$$

To order $d h / d \theta=0$, when $\theta=\alpha$, the maximum of $h$ can be solved

$$
h_{\max }=\frac{d}{2 \sin \alpha} e^{\alpha \tan \varphi} .
$$

Eq. (2) is the depth of concrete crater created by a flat-nose projectile.

\subsubsection{The slip line field crater created by a non-flat nose projectile}

Based on the slip line theory $[5,6]$, and the friction between the projectile and target is ignored, when the non-flat nose penetrate into the concrete, the target slip line filed is shown in Fig.2. Analogous to the flat nose projectile penetrate into concrete, the flowing plastic zone also can be divided into three regions, but the maximum crater depth of the concrete attaches to the projectile. Therefore, for arbitrary nose shape, it is assumed that the nose or projectile head immerses into the target, as shown in Fig. 2, which produces a crater depth of plastic or failure zone is equal to the nose height $H[3]$

$$
h_{\max }^{\prime}=H \text {. }
$$

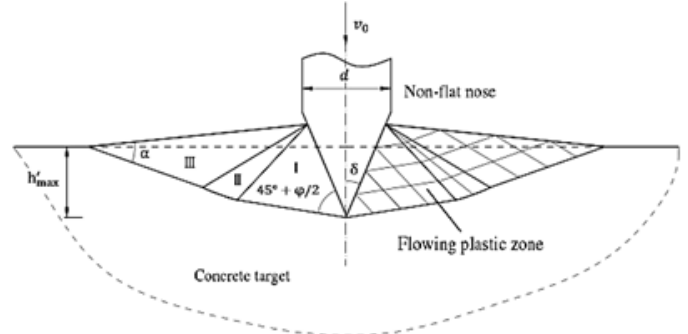

Fig.2 The slip line field of concrete crater created by a projectile with arbitrary nose.

\subsection{The crater depth of a normal projectile}

For a normal projectile with arbitrary nose shape, its crater process is the process of the sharp nose create the crater and then follow with flat nose, namely, the crater depth of the penetration can be simplified as the sum of the crater depth by arbitrary nose $h_{\max }^{\prime}$ and the crater depth by flat nose $\mathrm{h}_{\max }$ [3]. According to the formulas in this paper, the coefficient of crater depth $k$ can be shown as

$$
k=\frac{e^{\alpha \tan \varphi}}{2 \sin \alpha}+\frac{H}{d} .
$$


Obviously, if $\varphi=0$, then $k=0.707+H / d$, the result is just as the literature [3].

\section{The Effect of Impact Velocity on the Internal Friction Angle and Crater Depth}

There exists the strength dynamic effect of concrete in dynamic condition. Based on the Mohr-Coulomb theory and in static condition, the limit equilibrium condition on a point is shown as

$$
\sigma_{1 s}=\sigma_{3} \tan ^{2}\left(45^{\circ}+\frac{\varphi_{s}}{2}\right)+2 c_{s} \tan \left(45^{\circ}+\frac{\varphi_{s}}{2}\right) .
$$

Where $\varphi_{s}$ is the static internal friction angle and $\mathrm{c}_{\mathrm{s}}$ is the static cohesive strength.

In dynamic condition, strain rate will influence internal friction angle and cohesive strength. If the dynamic process is still consistent with the Mohr-Coulomb theory, and then the limit equilibrium condition can be shown as

$$
\sigma_{1 d}=\sigma_{3} \tan ^{2}\left(45^{\circ}+\frac{\varphi_{d}}{2}\right)+2 c_{d} \tan \left(45^{\circ}+\frac{\varphi_{d}}{2}\right) .
$$

Under the condition of uniaxial compression, namely, $\sigma_{3}=\sigma_{3 d}=0$, the relationship between the dynamic stress intensity factor and the internal friction angle, the cohesive strength can be established as

$$
\mathrm{K}=\frac{\sigma_{1 d}}{\sigma_{1 s}}=\frac{c_{d} \tan \left(45^{\circ}+\varphi_{d} / 2\right)}{c_{s} \tan \left(45^{\circ}+\varphi_{s} / 2\right)} .
$$

In general, the strength dynamic effect impact on the spall strength can be ignored, namely, the spall strength is a constant. And it can be shown as

$$
c_{s} \cot \varphi_{s}=c_{d} \cot \varphi_{d} \text {. }
$$

Based on the formula (7) and (8), the formula about the relationship between the dynamic stress intensity factor and the internal friction angle can be identified as

$$
\mathrm{K}=\frac{\sigma_{1 d}}{\sigma_{1 s}}=\frac{\tan \varphi_{d} \tan \left(45^{\circ}+\varphi_{d} / 2\right)}{\tan \varphi_{s} \tan \left(45^{\circ}+\varphi_{s} / 2\right)} .
$$

Considering the dynamic stress intensity factor formula of concrete by CEB [7], the formula about the relationship between the internal friction angle and the strain rate can be got

$$
\frac{\tan \varphi_{d} \tan \left(45^{\circ}+\varphi_{d} / 2\right)}{\tan \varphi_{s} \tan \left(45^{\circ}+\varphi_{s} / 2\right)}=m \dot{\varepsilon}^{\beta}, \dot{\varepsilon}>30 s^{-1},
$$

where $m$ and $\beta$ are the material constants.

When the projectile impacts the target, the strain rate of the concrete target is related to the projectile impact velocity. Base on the cavity expansion theory and considering the incompressible material, the target strain rate formula is deduced

$$
\dot{\varepsilon}=\frac{2 a^{2} \dot{a}}{r^{3}} \text {. }
$$

where $a$ and $\dot{a}$ are respectively the cavity radius and the cavity expansion rate. And the value of $\dot{a}$ is equal to the normal velocity of projectile surface.

Therefore, the formula about the relationship between the strain rate and impact velocity can be got, as shown in Eq. (12)

$$
\dot{\varepsilon}=\frac{2 V \sin \delta}{a}
$$

In Eq. (12) $\delta$ is the angle of the normal surface and axis of projectile and $V$ is the projectile velocity of the end of creates the crater, and because of the projectile resistance $V$ is small than impact velocity. 
The formula about the relationship between the internal friction angle and impact velocity can be determined by Eq. (10) and Eq. (12). And combined with Eq. (4), it can be found out that the crater depth is related to the impact velocity which has a main effect on the strain rate, and the internal friction angle will influence the crate depth with the increasing of strain rate.

\section{Test Verification}

\subsection{Test overview}

In this paper, the test verification is based on the static and dynamic mechanical behaviors research of UHMWPE (Ultra-high molecular weight polyethylene) fiber reinforced concrete and the penetration test of the same material in Ref. [9] and [10].

Primary materials: Ordinary P.O42.5 cement is used throughout the program. Coarse aggregate is size of 5 10 mm aggregate. River sand is with 2.5 fineness modulus. FDN-5 water reducer which can reduce water $26.5 \%$ and silica ash are also used. The UHMWPE fiber is the ZTX99-1200D supplied by the Hunan ZhongTai Special Equipment Co, Ltd, China.

Considering four fiber volume rates about $0 \%, 0.3 \%, 0.5 \%$ and $0.7 \%$ of the fiber reinforced concrete specimens, the verification test of the prism uniaxial and general tri-axial static compression test, the Hopkinson pressure bar dynamic compression test and penetration test [8,9] can be conducted. The specimen's size is $150 \times 150 \times 300 \mathrm{~mm}$ in the prism uniaxial compression test. The size of specimens is $\varphi 50 \times 100 \mathrm{~mm}$ and the 5 variations of confining pressure is $0 \mathrm{MPa}, 3 \mathrm{MPa}$, $6 \mathrm{MPa}, 12 \mathrm{MPa}$ and $18 \mathrm{MPa}$ in the general tri-axial test. The size of specimens is $\varphi 92 \times 46 \mathrm{~mm}$, considering 5 variations of strain rate which is $0 \mathrm{~s}^{-1}, 140 \mathrm{~s}^{-1}, 175 \mathrm{~s}^{-1}, 200 \mathrm{~s}^{-1}, 255 \mathrm{~s}^{-1}$ in the dynamic test. The specimens' size of the penetration test is $\varphi 500 \times 270 \mathrm{~mm}$, the projectile type is api-t $12.7 \mathrm{~mm}$, and the impact velocity is among $650 \sim 850 \mathrm{~m} / \mathrm{s}$.

\subsection{Determination calculation parameters}

According to this theory in the paper and based on the material property, firstly, the internal friction angle and the dynamic stress intensity factor parameters and so on should be determined.

Secondly, based on the Mohr-Coulomb theory, then the data of the tri-axial test could be processed and the parameters of the internal friction angle and cohesive strength can be solved, as shown in the Table 1and Fig. 3.

Table 1 The static internal friction angle of UHMWPE fiber reinforced concrete.

\begin{tabular}{ccccc}
\hline$V_{f} / \%$ & $a / M P a$ & $\tan \theta$ & $c / M P a$ & $\varphi_{s} /{ }^{\circ}$ \\
\hline 0 & 6.0733 & 0.7352 & 8.959 & 47.32 \\
0.3 & 7.9177 & 0.6196 & 10.087 & 38.29 \\
0.7 & 7.0779 & 0.6432 & 9.244 & 40.03 \\
\hline
\end{tabular}

Table 2 The results of dynamic effects parameters fitting.

\begin{tabular}{ccccc}
\hline$V_{f} / \%$ & 0 & 0.3 & 0.5 & 0.7 \\
\hline$m$ & 0.01516 & 0.05111 & 0.04000 & 0.01505 \\
$\beta$ & 0.8654 & 0.6206 & 0.6848 & 0.8587 \\
\hline
\end{tabular}

Table 2 and Fig. 4 show the fitted relation of different fiber volume rates in the formula of CEB. Based on Fig. 3 and Fig. 4, the variations of related parameters are reflected well in Table 1 and Table 2. 


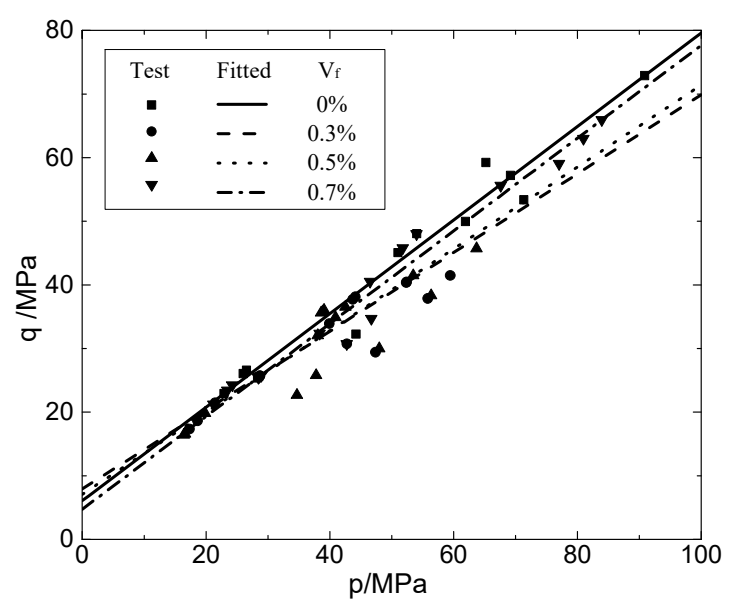

Fig.3 The stress path of specimen on general tri-axial test.

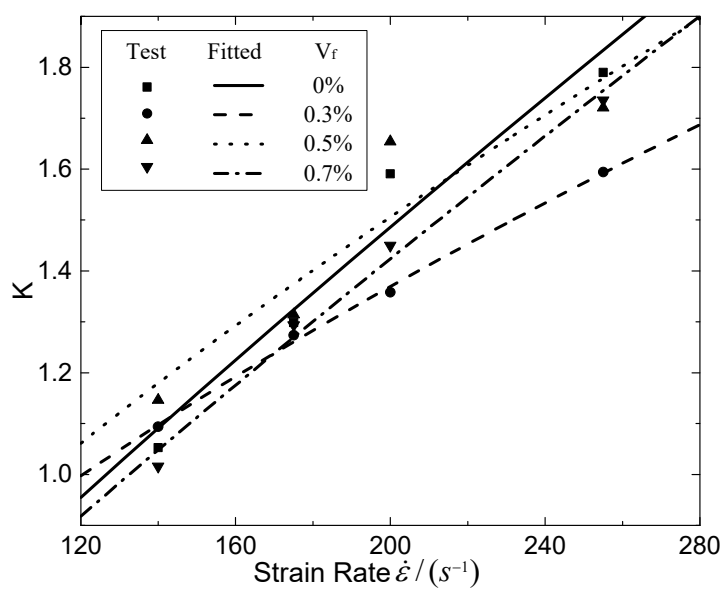

Fig.4 The dynamic effects parameters fitting.

\subsection{The Crater Depth Calculation and Verification}

\subsubsection{Verification ignoring the strain rates effect}

Base on the internal friction angle data in Table1, the crater depth is solved without the effect of strain rates, as shown in Table3. And the comparative data comes from Ref. [3] and [4]. The mean value of the crater depth and diameter in the test is used to calculate in the paper. And the parameter $H / d$ is 1.3733 and $\sin \delta$ is 0.1758 on the basis of the size of the projectile core.

Table 3 The calculation results of pit depth under static angle of internal friction.

\begin{tabular}{ccccccc}
\hline \multirow{2}{*}{$V_{f} \%$} & $V \mathrm{~m} / \mathrm{s}$ & $D \mathrm{~mm}$ & \multicolumn{5}{c}{$h / \mathrm{mm}$} \\
\cline { 4 - 7 } & 836 & 215 & The test data & In the paper & Ref.[3] & Ref. [4] \\
\hline \multirow{2}{*}{0} & 759 & 206 & 33 & & & 528.8 \\
& 642 & 164 & 26 & 25.7 & 15.6 & 491.6 \\
0.3 & 835 & 123 & 41 & & & 433.3 \\
& 759 & 157 & 29 & 22.6 & 15.6 & 528.9 \\
& 650 & 112 & 26 & & & 492.2 \\
0.5 & 842 & 150 & 40 & & & 547.9 \\
& 751 & 141 & 34 & 23.1 & 15.6 & 499.6 \\
& 642 & 110 & 30 & & & 443.9 \\
0.7 & 845 & 136 & 35 & & & 541.7 \\
& 758 & 113 & 29 & 25.5 & 15.6 & 499.1 \\
& 653 & 107 & 23 & & & 446.0 \\
\hline
\end{tabular}

From Table 3, when the internal friction angle is static, it is known that the calculation result can be well consistent with the low speed $(642-653 \mathrm{~m} / \mathrm{s})$, however, there is a larger deviation for the medium and high speed $(751-759,835-845 \mathrm{~m} / \mathrm{s})$ in the test. The result in Ref. [3] is a fixed value $1.56 \mathrm{~mm}$ which only relates to the bullet shape, and it is lower than the test result obviously. The calculation value in Ref. [4] is much larger than the test value, but it can be discovered that the calculation value is similar to the change rule of the test result with the increase of impact velocity, so the effect of the velocity on the crater depth should be considered.

\subsubsection{Verification considering the strain rate effect}

When the projectile is creating a crater, the crater can be regard as a part of the cavity. Then the cavity radius can be estimated and it is about ten times projectile diameter by the Table 3 . Based on the strain rate effect model, the relationship between the internal friction angle, the crater depth and the impact velocity can be determined, as shown in Table4 and Fig. 5.

As shown in Table 4 and Fig.5, it can be found:

(1)The corrected calculation result of the crater depth can reflect the change rule about the depth varying with impact velocity, and compared with the value ignoring the dynamic internal friction angle effect, the result can be consistent with the test data, the deviation is lower. 
Table 4 The calculation results of pit depth under dynamic angle of internal friction.

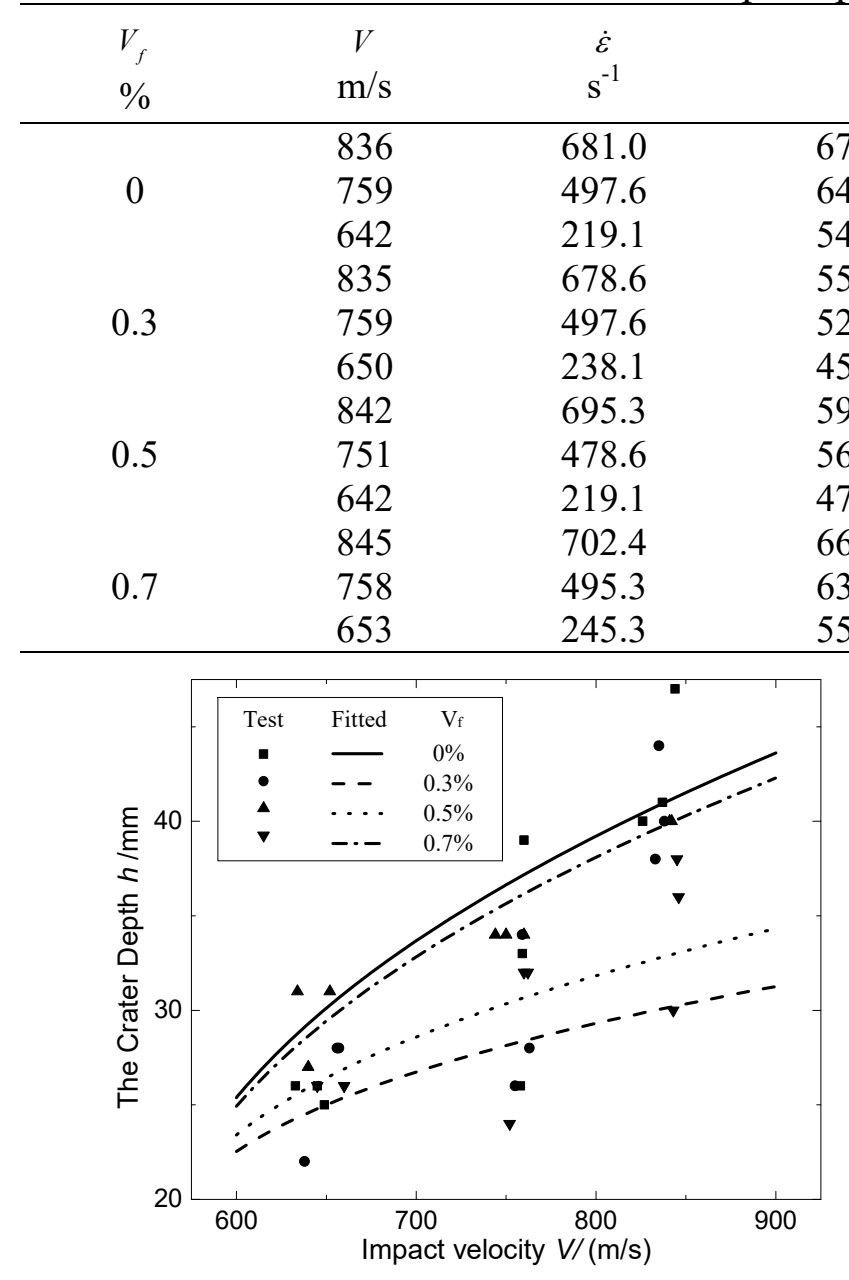

(a) The crater depth \& impact velocity

\begin{tabular}{cccc}
\multirow{2}{*}{$\varphi$} & \multicolumn{2}{c}{$h / \mathrm{mm}$} & relative \\
\cline { 2 - 3 } & The test data & $\begin{array}{c}\text { Modification } \\
\text { value }\end{array}$ & error \\
\hline 67.30 & 43 & 40.496 & 0.06 \\
64.26 & 33 & 37.127 & 0.13 \\
54.78 & 26 & 29.457 & 0.13 \\
55.73 & 41 & 30.043 & 0.27 \\
52.85 & 29 & 28.359 & 0.02 \\
45.49 & 26 & 24.999 & 0.04 \\
59.81 & 40 & 32.948 & 0.18 \\
56.25 & 34 & 30.376 & 0.11 \\
47.98 & 30 & 26.012 & 0.13 \\
66.70 & 35 & 40.082 & 0.15 \\
63.25 & 29 & 36.063 & 0.24 \\
55.11 & 23 & 29.659 & 0.29 \\
\hline
\end{tabular}

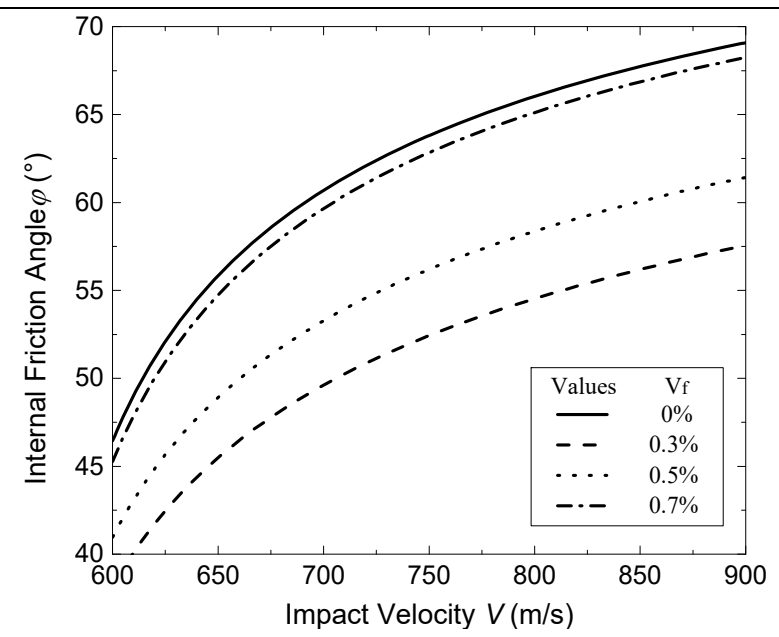

(b) The internal friction angle \& impact velocity

Fig.5 Variation of pit depth and internal friction angle with impact velocity.

(2)The crater depth of rigid-plastic projectile penetration concrete will be deeper as the increasing internal friction angle. The internal friction angle will be larger with the increasing impact velocity, and with the increase of impact velocity, the increase rate is reducing.

(3)With the velocity increasing, the increase speed of fiber reinforced concrete internal friction angle will decrease with the fiber volume fraction increasing. It is shown that the increase of fiber content will cause the strain rate to decrease, namely, the strain rate sensitivity will be higher with the fiber content is lower, which is consistent with Ref. [10].

\section{Conclusions}

(1)Based on the slip line theory, the crater depth formula of rigid-plastic projectile penetration concrete considering internal friction angle is established, and the formula is well matched with the low impact velocity in the test.

(2)The relationship between the internal friction angle, the strain rate and the impact velocity can be obtained by the static and dynamic performance test of concrete, and the formula of the three variables can be established. Then the calculation values of formulas are compared with the penetration test result of UHMWPE fiber reinforced concrete, and it is proved that these formula have good applicability.

(3)The crater depth of rigid-plastic projectile penetration concrete is related to the impact velocity on the surface, however, essentially the different impact velocity will lead to the different strain rate, then the internal friction angle will change with the different strain rate. The result shows that the crater depth will increase with the increase of internal friction angle. 


\section{References}

[1] M.J. Forrestal, B.S. Altman, J.D. Cargile, S.J. Hanchak: International Journal of Impact Engineering, Vol. 15 (1994) No.4, p.359

[2] D.J. Frew, S.J. Hanchak, M.L. Green, M.J. Forrestal: International Journal of Impact Engineering, Vol. 21 (1998), p.489

[3] Q.M. Li ,X.W. Chen: International Journal of Impact Engineering, Vol. 28(2003), p.93

[4] Z.P. Wen, Y.X. Wang, B.M. Lv: Journal of Changzhou Institute of Technology, Vol. 18 (2005)

No. E, p.82 (in Chinese)

[5] Z.Y. Liu: Soil Mechanics (China Electric Power Press, China 2007), p.162

[6] X. Zhang, G.X. Wu: Engineering Plasticity Theory (Beihang University Press, China 1998), p. 244

[7] Code, CEB-FIP Model: Bulletin D'Information, Vol.117(1990) No. E

[8] Q.H. Qian, M.Y. Wang: Calculation Theory for Advanced Protective Structures (Jiangsu Science and Technology Press, China 2009), p.39

[9] L.F. Li: Experimental Research on Mechanical Behaviors and Anti-penetration Properties of UHMWPE Fiber Reinforced Concrete (MS.,National University of Defense Technology, China 2015), p.10

[10]Y.W. Zhang: Research on Static and Dynamic Mechanical Behaviors of UHMWPE Fiber Reinforced Concrete (MS.,National University of Defense Technology ,China 2014 ), p.22 